\title{
Thermal radiation from magnetic neutron star surfaces
}

\author{
J. F. Pérez-Azorín, J. A. Miralles, and J. A. Pons
}

Departament de Física Aplicada, Universitat d'Alacant, Ap. Correus 99, 03080 Alacant, Spain

Received 7 July 2004 / Accepted 22 November 2004

\begin{abstract}
We investigate the thermal emission from magnetic neutron star surfaces in which the cohesive effects of the magnetic field have produced the condensation of the atmosphere and the external layers. This may happen for sufficiently cool $(T \leq$ $10^{6}$ ) atmospheres with moderately intense magnetic fields (about $10^{13} \mathrm{G}$ for Fe atmospheres). The thermal emission from an isothermal bare surface of a neutron star shows no remarkable spectral features, but it is significantly depressed at energies below some threshold energy. However, since the thermal conductivity is very different in the normal and parallel directions to the magnetic field lines, the presence of the magnetic field is expected to produce a highly anisotropic temperature distribution, depending on the magnetic field geometry. In this case the observed flux of such an object looks very similar to a BB spectrum, but depressed by a nearly constant factor at all energies. This results in a systematic underestimation of the area of the emitter (and therefore its size) by a factor 5-10 (2-3).
\end{abstract}

Key words. stars: neutron - radiation mechanisms: thermal - X-rays: stars

\section{Introduction}

Almost a decade after the discovery of the soft X-ray source RX J185635-3754 (Walter et al. 1996) using the ROSATPSPC, the thermal component associated with the direct emission from a neutron star's surface has been detected in more than $20 \mathrm{X}$-ray sources. In many cases it is superimposed on a power-law tail, but seven of these objects are wellcharacterized as simple blackbodies with temperatures ranging between 60 and $100 \mathrm{eV}$. The apparent small emitting surface of RX J185635-3754, inferred from the best blackbody fit and its parallax (Walter \& Lattimer 2002) have led to speculation about its nature, and whether its apparent smallness can be considered as evidence that the object is a strange star: a self-bound object made of up, down and strange quarks (Pons et al. 2002; Drake et al. 2002), or a standard, misaligned pulsar. Although this new observational class (isolated compact stars) is probably the first real opportunity to place stringent constraints on the equation of state (EOS) of dense matter from astrophysical measurements (see Lattimer \& Prakash 2001, for a review), one must be cautious before concluding that an apparently small X-ray source is a quark star, because the $\mathrm{X}$-ray spectrum is not the only information available. Using the Hubble Space Telescope, Walter \& Matthews (1997) subsequently identified an optical source at $6060 \AA$ and $3000 \AA$, with a brightness only about 7 times brighter than an extrapolation of a $62 \mathrm{eV}$ X-ray blackbody into the optical $V$ band. The observed optical fluxes have been confirmed by subsequent observations from the 2-m NTT (Neuhaüser et al. 1998) and new HST measurements (Pons et al. 2002). Remarkably, the other three isolated compact X-ray sources that have been detected in the optical band (RX J0720.4-3125, RX J1308.6+2127, and RX J1605.3+3249) also have a significant optical excess over the extrapolation of the X-ray blackbody (a factor 5 to 14). None of them have yet been detected as $1.4 \mathrm{GHz}$ radio sources. Thus, RX J185635-3754 is not an uncommon object, for it shares the same general observational properties of other isolated neutron stars (blackbody spectrum in X-ray, no evident spectral features, optical excess), except for the fact that four of them have fairly long periods (8-22 s), while RX J185635-3754 is not variable, with a reported upper limit on the pulse amplitude of $<1.3 \%$ (Burwitz et al. 2003).

Since blackbodies are no more than a simple approximation of the true emission mechanism, a step forward in understanding the thermal emission of neutron stars, consists of computing model atmospheres for low magnetic fields $\left(<10^{11} \mathrm{G}\right)$ and emergent spectra for several compositions, masses and radius (Romani 1987; Miller 1992; Rajagopal \& Romani 1996). The parameters that determine the shape of the observed spectrum are the atmospheric composition, the red-shifted temperature, $T_{\infty}=T_{\text {eff }} /(1+z)$, the redshift $z$, the interstellar medium absorption, $n_{H}$, and the ratio $R_{\infty} / d$, where $d$ is the distance to the object and $R_{\infty}=R(1+z), R$ being the radius of the star. For a Planck spectrum the redshift factors contribute only to an overall scale factor, so that the redshift does not affect the models. For realistic atmospheres with spectral features, however, the redshift is an important parameter because the identification of spectral lines would determine the $M / R$ ratio and establish relevant constraints on the EOS of dense matter. After a detailed investigation of the different parameters that affect the observed spectra, Pons et al. (2002) concluded that X-ray data alone do not allow us to establish severe constraints, given the large number of degrees of freedom. The combination of the X-ray spectra with the optical fluxes turned 
out to be much more restrictive, as was suggested earlier by Pavlov et al. (1996). The broadband spectral energy distribution (SED) of isothermal heavy element atmospheres can fit the observed SED, but these models predict detectable absorption lines and edges. It was hoped that the more sensitive spectrographs on Chandra and XMM-Newton would be able to detect spectral features and help to understand the nature of this objects, but no lines or absorption edges have been seen in long exposures (Burwitz et al. 2001; Drake et al. 2002). Only the presence of broadband departures from a single blackbody spectrum has recently been suggested (Burwitz et al. 2003).

The puzzle, then, is as follows: single temperature models based on heavy element atmospheres explain the broadband spectrum and the lack of variability of the X-ray spectrum, but they are in contradiction with the absence of spectral features. Single blackbody models, besides being unrealistic, cannot account for the systematic optical excess that is observed in 4 sources. An anisotropic surface temperature distribution (the simplest case would be two blackbody components) can reconcile X-ray and optical observations, because the quantity $\left(R_{\infty} / D\right)^{2}$ must be interpreted as the solid angle subtended by the star's surface area which is visible at some distance $D$. Thus the true value of $R_{\infty}$ will generally be larger if the assumption that the temperature is uniform on the surface is relaxed. However, this is barely consistent with the lack of pulsation of RX J185635-3754, unless its magnetic and rotation axes are oriented in a very particular way.

Nevertheless, there is an important ingredient missing in the above discussion, the likely existence of strong magnetic fields. The strong $\left(10^{12-13} \mathrm{G}\right)$ magnetic fields of pulsars suggest that most, if not all, neutron stars should have similarly strong fields. However, detailed atmosphere models with strong magnetic fields are only available for hydrogen (Zavlin et al. 1995), because reliable opacities and EOS have not yet been developed for heavier elements and because of the complexity of modeling magnetic atmospheres with arbitrary magnetic field structures. For heavy-element dominated atmospheres, only approximate treatments of magnetic $\mathrm{Fe}$ atmospheres exist (Rajagopal et al. 1997), and the results show that the spectra are globally much closer to a blackbody than for light element atmospheres. There is a case to be made that RX J185635-3754 is magnetized. The deep VLT image of the target released by van Kerkwijk \& Kulkarni (2000) shows what looks like a classic bow-shock nebula. The presence of a bow-shock suggests that this is a magnetized neutron star with a relativistic wind, as seen in the pulsars PSR 1957+20 (Kulkarni \& Hester 1988) and PSR 2224+65 (Cordes et al. 1993).

An alternative possibility that explains naturally the absence of spectral features is the emission from a solid surface. This was a common idea 20-30 years ago (Brinkmann 1980, B80 in the following) until the existence of a thin gaseous atmosphere was appreciated and model atmospheres became more popular. However, at sufficiently low temperatures, highly magnetized neutron stars may undergo a phase transition that turns the gaseous atmosphere into a solid (Lai 2001). The critical temperature below which the atmosphere condensates depends on the composition and the magnetic field. For example, for typical magnetic field strengths of $10^{13} \mathrm{G}$, a Fe atmosphere will condensate for $T<0.1 \mathrm{keV}$ while a $\mathrm{H}$ atmosphere needs temperatures lower than $0.03 \mathrm{keV}$ to undergo the phase transition to the metallic state (Lai 2001). Notice that effective temperatures of the observed isolated neutron stars fall in this temperature range, therefore they should plausibly be in the solid state if the dominant element in the atmosphere is Fe. In such a metallic neutron star surface made of nuclei with atomic number $Z$ and atomic weight $A$, the pressure vanishes at a finite density

$\rho_{s} \approx 560 A Z^{-3 / 5} B_{12}^{6 / 5} \mathrm{~g} \mathrm{~cm}^{-3}$

where $B_{12}$ is the magnetic field in units of $10^{12} \mathrm{G}$.

This idea has been recently revisited by Turolla et al. (2004), who found that the emitted spectrum is strongly depressed at low energies, thus making more difficult the reconciliation between observational data and the condensed surface model. However, in this last work the emissivity is calculated neglecting one of the transmission modes in some cases. This simplification is inaccurate and can modify the emitted spectrum, as we will discuss in the text. Besides, the sole presence of a strong magnetic field, necessary to condensate the atmosphere, results naturally in an anisotropic surface temperature (Geppert et al. 2004), which must be calculated consistently with the magnetic field structure. In this paper our aim is to study the thermal emission from solid surfaces of neutron stars and its implications on the observational properties of isolated compact objects.

The paper is organized as follows: Sect. 2 is devoted to discussing the calculation of the emissivity starting from the description of the dielectric tensor for the condensed neutron star surface. In Sect. 3 we show results, including integrated spectra for different strengths and geometries of the magnetic field. Final remarks and a summary of the main conclusions are discussed in Sect. 4.

\section{Equations and input microphysics}

\subsection{Dielectric tensor}

The emission properties (reflectivity, emissivity) of a neutron star surface in a condensed state are obtained from the dielectric tensor, and were first studied in detail in B80. In the presence of the strong magnetic fields expected to exist at the surface of a neutron star, the dielectric tensor changes significantly with respect to the non-magnetic case, leading to birefringence and other associated phenomena. In order to calculate the dielectric tensor in the presence of magnetic fields and to take into account dissipative processes, it is better to obtain first the conductivity tensor $\sigma_{i j}$, and then to calculate the dielectric tensor $\epsilon_{i j}$ according to the equation

$\epsilon_{i j}=\delta_{i j}+\mathrm{i} \frac{4 \pi}{\omega} \sigma_{i j}$

In this expression, $\omega$ is the angular frequency of the electromagnetic wave propagating in the medium.

For a magnetized plasma, electrical conductivities for the static case $(\omega=0)$ have been calculated by some authors (Hernquist 1984; Potekhin 1999) with and without taking into 
account the quantizing effect of the magnetic field. The general expression for the conductivity tensor can be written as follows

$\sigma_{i j}=\int e^{2} \frac{\mathcal{N}_{B}}{\epsilon / c^{2}} \tau_{i j}\left(-\frac{\partial f_{0}}{\partial \epsilon}\right) \mathrm{d} \epsilon$

where $\epsilon$ is the electron energy, $f_{0}$ the Fermi-Dirac distribution, $\mathcal{N}_{B}$ is given by the expression (see Potekhin 1999, for details)

$\mathcal{N}_{B}=\frac{m_{\mathrm{e}} \omega_{B}}{2(\pi \hbar)^{2}} \sum_{n=0}^{n_{\max }} g_{n} \sqrt{(\epsilon / c)^{2}-\left(m_{\mathrm{e}} c\right)^{2}-2 m_{\mathrm{e}} \hbar \omega_{B} n}$

and the functions $\tau_{i j}$ play the role of effective relaxation times. For a non-quantizing magnetic field, these effective relaxation times can be expressed in terms of a relaxation time, $\tau_{0}$, which is the inverse of the collisional frequency $v^{D}$ (D after damping) However, in the quantizing case, collisional frequencies in the longitudinal $v_{\|}^{D}$ and perpendicular $v_{\perp}^{D}$ direction to the magnetic field are no longer equal. For a quantizing magnetic field, the components of the $\tau_{i j}$ tensor are

$\tau_{z z}=\tau_{\|} ; \quad \tau_{x x}=\frac{\tau_{\perp}}{1+\left(\omega_{B} \tau_{\perp}\right)^{2}} ; \quad \tau_{y x}=\frac{\omega_{B} \tau_{\perp}^{2}}{1+\left(\omega_{B} \tau_{\perp}\right)^{2}}$

with $\tau_{\|}$and $\tau_{\perp}$ being the inverse of the effective collisional frequencies, $v_{\|}^{D}$ and $v_{\perp}^{D}$, respectively, and $\omega_{B}=\frac{\mathrm{e} B}{m_{\mathrm{e}} c}$ being the electron cyclotron frequency.

In the dynamic case $(\omega \neq 0)$, the conductivity tensor can be obtained by the transformation

$\tau_{\perp}^{-1} \rightarrow \tau_{\perp}^{-1}-\mathrm{i} \omega$

$\tau_{\|}^{-1} \rightarrow \tau_{\|}^{-1}-\mathrm{i} \omega$

and the dielectric tensor, for degenerate non-relativistic electrons, reads

$\epsilon_{i j}=\delta_{i j}+\mathrm{i} \frac{4 \pi}{\omega} \sigma_{i j}=\left(\begin{array}{ccc}S & -\mathrm{i} D & 0 \\ \mathrm{i} D & S & 0 \\ 0 & 0 & P\end{array}\right)$,

where we have defined the following complex quantities

$$
\begin{aligned}
\left(\begin{array}{l}
R \\
L
\end{array}\right) & =1-\frac{\omega_{\mathrm{p}}^{2}}{\omega^{2}} \frac{\omega}{\omega \mp \omega_{B}+\mathrm{i} v_{\perp}^{D}} ; \\
P & =1-\frac{\omega_{\mathrm{p}}^{2}}{\omega^{2}+\mathrm{i} \omega v_{\|}^{D}} ; \\
S & =\frac{1}{2}(R+L) ; \\
D & =\frac{1}{2}(R-L) .
\end{aligned}
$$

Here, $\omega_{\mathrm{p}}=\left(4 \pi \mathrm{e}^{2} n_{\mathrm{e}} / m_{\mathrm{e}}\right)^{1 / 2}$ is the electron plasma frequency and, using the density at zero pressure given by Eq. (1), the electron particle density $n_{\mathrm{e}}$ can be calculated as follows

$n_{\mathrm{e}}=1.24 \times 10^{27} Z_{26}^{2 / 5} B_{12}^{6 / 5} \mathrm{~cm}^{-3}$.

\subsection{The dispersion relation}

Consider an electromagnetic wave propagating in a medium described by a dielectric tensor $\epsilon_{\alpha \beta}$. The dispersion relation is easily obtained by introducing the Maxwell tensor

$\Lambda_{i j}=k_{i} k_{j}-k^{2} \delta_{i j}+\frac{\omega^{2}}{c^{2}} \epsilon_{i j}$,

where $k_{i}$ are the Cartesian components of the wave vector. Since, in terms of the Maxwell or dispersion tensor, the electric field of the wave satisfies the equation

$\Lambda_{i j} E_{j}=0$,

the condition to have a non-trivial solution leads to the dispersion equation

$\left|\Lambda_{i j}\right|=0$.

In the same Cartesian frame as used in B80, where the magnetic field $\boldsymbol{B}$ is in the $x-z$ plane and forms an angle $\alpha$ with the $z$-axis, which is normal to the surface, the dielectric tensor, obtained by a rotation transformation from Eq. (8), adopts the form

$\epsilon_{i j}=\left(\begin{array}{ccc}S \cos ^{2} \alpha+P \sin ^{2} \alpha & -\mathrm{i} D \cos \alpha & \sin \alpha \cos \alpha(P-S) \\ \mathrm{i} D \cos \alpha & S & -\mathrm{i} D \sin \alpha \\ \sin \alpha \cos \alpha(P-S) & \mathrm{i} D \sin \alpha & P \cos ^{2} \alpha+S \sin ^{2} \alpha\end{array}\right)$.

For our purposes it is convenient to write the dispersion relation for the transmitted wave in terms of the incident one. We consider an incident wave with wave vector

$\boldsymbol{k}_{i}=k_{i}(-\sin i \cos \beta, \sin i \sin \beta,-\cos i)$,

where $i$ is the angle of incidence and $\beta$ the azimuth. Since the wave is propagating in vacuum and we are neglecting vacuum polarization, the dispersion relation gives $k_{i}=\omega / c$. The reflected and transmitted waves have wave-vectors

$\boldsymbol{k}_{r}=k_{i}(-\sin i \cos \beta, \sin i \sin \beta, \cos i)$

$\boldsymbol{k}_{t}=k_{t}\left(-\sin \theta_{m} \cos \beta, \sin \theta_{m} \sin \beta,-\cos \theta_{m}\right) ; \quad m=1,2$,

where the subscript $m$ refers to the ordinary and extraordinary modes and $\theta_{m}$ is the angle of refraction, which is a complex number. Introducing the complex refractive index for the transmitted wave $n=k c / \omega$, and using the Snell law, $\sin \theta_{m}=$ $\sin i / n_{m}$, the dispersion relation leads to a quartic equation for the refractive index $n$,

$$
\begin{array}{r}
n^{4}\left(P+v \sin ^{2} \alpha\right)+n^{2}\left(g v-2 P S+u \sin ^{2} \alpha\right)+P R L+g u= \\
\sin i \sin (2 \alpha) \cos \beta\left(n^{2}-\sin ^{2} i\right)^{1 / 2}\left(u+n^{2} v\right)
\end{array}
$$

where $v=S-P, u=P S-R L$, and $g=\sin ^{2} i[1-$ $\left.\sin ^{2} \alpha\left(1+\cos ^{2} \beta\right)\right]$. This quartic equation can be solved analytically, after squaring, and the refractive indexes for both ordinary and extraordinary modes can be obtained after analyzing which are the two physical roots that satisfy the original Eq. (20).

In Fig. 1 we show the real and imaginary parts of the two modes as a function of the photon energy and for two different magnetic fields $B=5 \times 10^{12} \mathrm{G}$ (solid lines) and $B=5 \times 10^{13} \mathrm{G}$ (dashes). The corresponding electron plasma frequencies are 

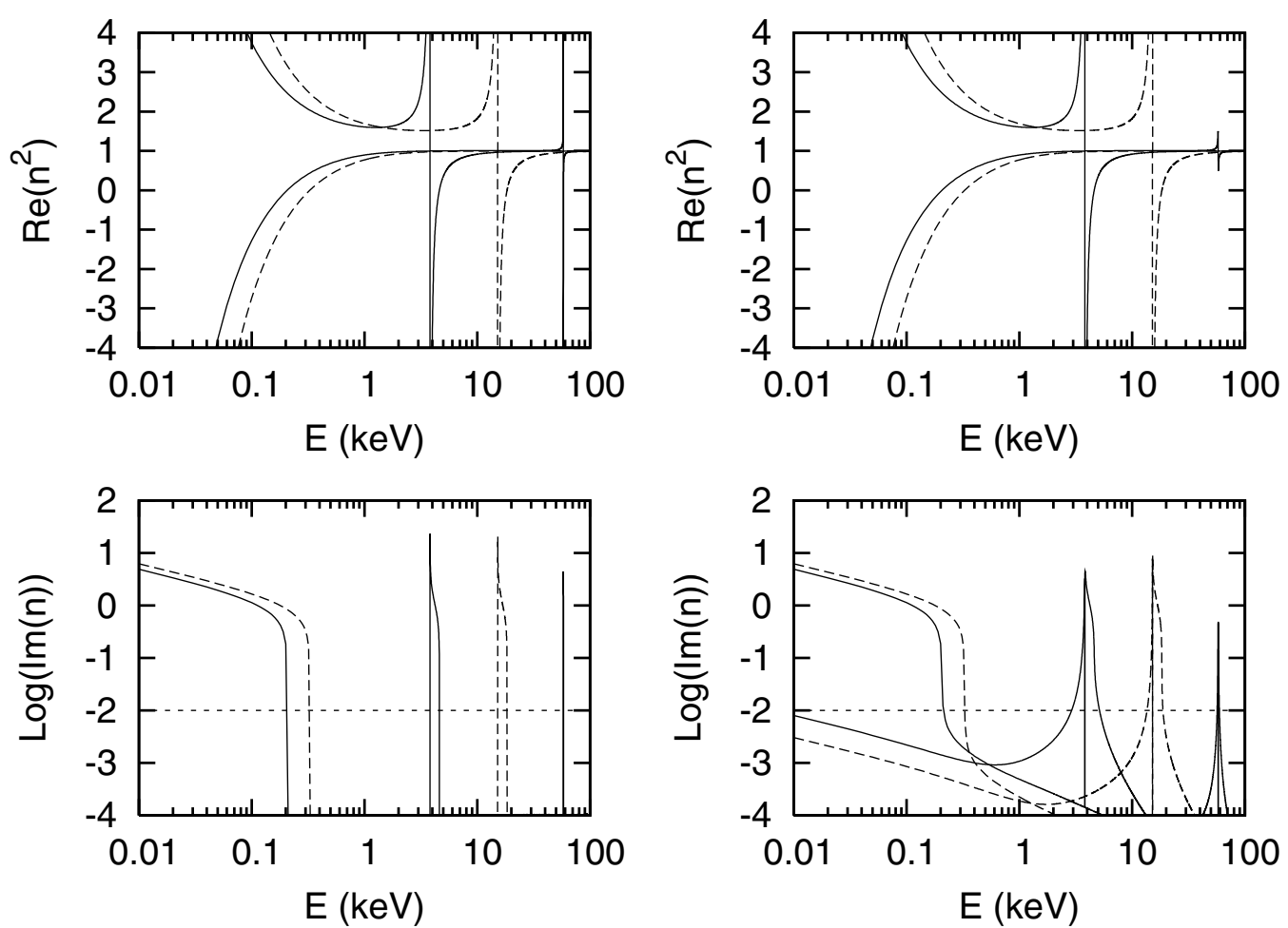

Fig. 1. Refractive indexes without (left) and with (right) damping effects. The real parts are shown in the top panels and the imaginary parts are shown at the bottom. Two different values of the magnetic field are depicted, $5 \times 10^{12} \mathrm{G}$ (solid lines) and $5 \times 10^{13} \mathrm{G}$ (dashed lines). The photon incidence angle has been taken to $43^{\circ}$ to compare with Fig. 6 of Turolla et al. (2004).

$3.6 \mathrm{keV}$ and $14.3 \mathrm{keV}$, respectively. We have taken a magnetic field normal to the emitting surface and an incidence angle of $43^{\circ}$. The left panels correspond to the case without damping $\left(v_{\|}^{D}=v_{\perp}^{D}=0\right)$ while the right panels show the equivalent results including effects of collisional damping (these collisional damping frequencies were calculated by using the public code developed by Potekhin ${ }^{1}$ ). The resonance at the plasma frequency $\omega_{p}$ is clearly visible, as well as a second, narrow resonance at $\omega_{B}\left(\approx 58 \mathrm{keV}\right.$ for $\left.B=5 \times 10^{12} \mathrm{G}\right)$. More interestingly, we can observe a region at low energy in which one of the modes takes a large imaginary part, which will result in significant absorption. The energy at which this happens can be estimated as follows. For simplicity, let us assume normal incidence and a magnetic field normal to the surface, and let us consider the case without damping. In this limit the dispersion relation is

$P n^{4}-2 P S n^{2}+P R L=0$,

and the roots are simply $n^{2}=L$ and $n^{2}=R$, i.e.,

$n^{2}=1-\frac{\omega_{p}^{2}}{\omega\left(\omega \pm \omega_{B}\right)}$.

Therefore, for energies $\omega<\frac{\omega_{B}}{2}\left[-1+\sqrt{1+4\left(\omega_{\mathrm{p}} / \omega_{B}\right)^{2}}\right]$ the second mode acquires an increasingly larger imaginary part. It turns out that for $B>10^{12} \mathrm{G}$ we are always in the case $\omega_{\mathrm{p}} \ll \omega_{B}$, and the above condition becomes approximately $\omega<\omega_{\mathrm{p}}^{2} / \omega_{B}$. In brief, we can expect two main features in the spectrum, a resonant absorption near the plasma and cyclotron

\footnotetext{
${ }^{1}$ www.ioffe.rssi.ru/astro/conduct/condmag.html
}

frequencies, and lower emission (compared to the $\mathrm{BB}$ ) below a certain threshold energy $\approx \omega_{\mathrm{p}}^{2} / \omega_{B}$. In Turolla et al. (2004), the modes with imaginary part larger than a certain value $(0.01)$ were neglected, this is indicated in the figure by the horizontal dashed line. In some cases, this may result in lower emissivity at low frequencies, and we prefer not to neglect them.

\subsection{Reflectivity and emissivity}

Knowing the complex refractive indices of the two modes, and solving Eq. (15), the ratio between the electric field components, $\left(E_{x}^{\prime}, E_{y}^{\prime}, E_{x}^{\prime}\right)$, of the transmitted wave can be obtained:

$$
\begin{aligned}
& \frac{E_{m, x}^{\prime}}{E_{m, z}^{\prime}} \equiv a_{m}=\left(D^{2} \sin ^{2} \alpha+S \sin ^{2} i-n_{m}^{2} \sin ^{2} i \cos ^{2} \beta\right. \\
& \left.\quad+\left(P \cos ^{2} \alpha+S \sin ^{2} \alpha\right)\left(n_{m}^{2}-S-\sin ^{2} i \sin ^{2} \beta\right)\right) \\
& \quad \times\left(\left[\sin i \cos \beta \sqrt{n_{m}^{2}-\sin ^{2} i}+\sin \alpha \cos \alpha(P-S)\right]\left(S-n_{m}^{2}\right)\right. \\
& \quad+\mathrm{i} D \sin i \sin \beta\left(\cos \alpha \sqrt{n_{m}^{2}-\sin ^{2} i}+\sin \alpha \sin i \cos \beta\right) \\
& \left.\quad+\sin \alpha \cos \alpha\left[(P-S) \sin ^{2} i \sin ^{2} \beta+D^{2}\right]\right)^{-1}
\end{aligned}
$$

and

$$
\begin{aligned}
\frac{E_{m, y}^{\prime}}{E_{m, z}^{\prime}} \equiv & b_{m}=\left[a_{m}\left(\sin ^{2} i \cos \beta \sin \beta-i D \cos \alpha\right)\right. \\
& \left.+\sin \beta \sin i \sqrt{n_{m}^{2}-\sin ^{2} i}+i D \sin \alpha\right] \\
& \times\left(\sin ^{2} \beta \sin ^{2} i-n_{m}^{2}+S\right)^{-1}
\end{aligned}
$$


These relative amplitudes coincide, after some minor algebraic manipulation, with those in B80. In Turolla et al. (2004), a different linear combination of the three equations arising from Eq. (15) was used, but both results can be shown to be equivalent when the refraction index is a solution of Eq. (20).

The boundary conditions at the surface of separation of both media, (vacuum and the magnetized plasma) imply that the tangential component of the magnetic and electric field and the normal component of the magnetic and the electric displacement must be continuous. This results in the following equation

$\left(\begin{array}{c}E_{\perp} \\ E_{\perp} \\ E_{\|} \\ E_{\|}\end{array}\right)=\left(\begin{array}{cccc}B_{1} & B_{2} & -1 & 0 \\ \frac{n_{1} \cos \theta_{1} B_{1}}{\cos i} & \frac{n_{2} \cos \theta_{2} B_{2}}{\cos i} & 1 & 0 \\ \frac{A_{1}}{\cos i} & \frac{A_{2}}{\cos i} & 0 & 1 \\ \frac{C_{1}}{\sin i} & \frac{C_{2}}{\sin i} & 0 & -1\end{array}\right) \cdot\left(\begin{array}{c}E_{1 z}^{\prime} \\ E_{2 z}^{\prime} \\ E_{\perp}^{\prime \prime} \\ E_{\|}^{\prime \prime}\end{array}\right)$

where $E, E^{\prime}$ and $E^{\prime \prime}$ are, respectively, the electric field of the incident, transmitted and reflected wave, and the subscripts $\|$, $\perp$ refer to components parallel or perpendicular to the incidence plane. Above, we have defined $A_{m}=b_{m} \sin \beta-a_{m} \cos \beta$, $B_{m}=b_{m} \cos \beta+a_{m} \sin \beta$, and $C_{m}=\epsilon_{31} a_{m}+\epsilon_{32} b_{m}+\epsilon_{33}$. This system of equations can be solved for the electric field of the reflected wave in terms of the incident one. Details and explicit expressions are given in the Appendix.

The reflectivity can be now obtained by assuming that the incident wave is the incoherent sum of two linearly polarized waves parallel and perpendicular to the incidence plane. From Eq. (A.1) and taking $E_{\perp}=1, E_{\|}=0$, the two complex components of the reflected field $\left(E_{\|}^{\prime \prime}\right.$ and $\left.E_{\perp}^{\prime \prime}\right)$ can be calculated, and the reflectivity is simply

$\rho_{\perp}=\left|E_{\|}^{\prime \prime}\right|^{2}+\left|E_{\perp}^{\prime \prime}\right|^{2}$

Analogously, for the case of polarization in the incidence plane, one takes $E_{\perp}=0, E_{\|}=1$, and after obtaining $E_{\|}^{\prime \prime}$ and $E_{\perp}^{\prime \prime}$ the reflectivity is given by

$\rho_{\|}=\left|E_{\|}^{\prime \prime}\right|^{2}+\left|E_{\perp}^{\prime \prime}\right|^{2}$.

To obtain the reflectivity of the unpolarized incident, $\rho_{v}$, wave we just take the average

$\rho_{v}=\frac{1}{2}\left(\rho_{\perp}+\rho_{\|}\right)$.

Finally, the reflectivity is related to the emitted specific intensity $I_{v}$ by Kirchhoff's law

$I_{v}=\left(1-\rho_{v}\right) B_{v}$,

where $B_{v}$ is the Planck intensity

$B_{v}=\frac{2 h v^{3} / c^{2}}{\exp (h v / k T)-1}$.

In Fig. 2 we illustrate how the normalized emissivity, i.e., emitted intensity normalized to the BB value, $\alpha_{v} \equiv I_{v} / B_{v}=1-\rho_{v}$, varies with the angle of incidence for a fixed magnetic field of $B=10^{12} \mathrm{G}$ normal to the surface. The temperature is $T=10^{6} \mathrm{~K}$. The emissivity is strongly reduced compared to

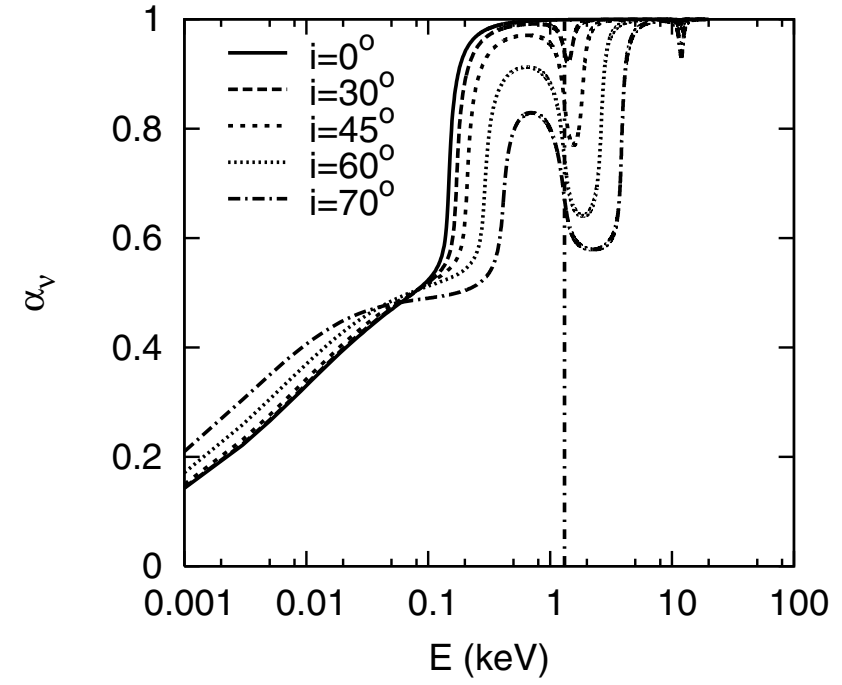

Fig. 2. Emissivity normalized to the BB emission as a function of energy for $B=10^{12} \mathrm{G}$ and $T=10^{6} \mathrm{~K}$ and varying the incident angle. The magnetic field has been taken to be normal to the emitting surface.

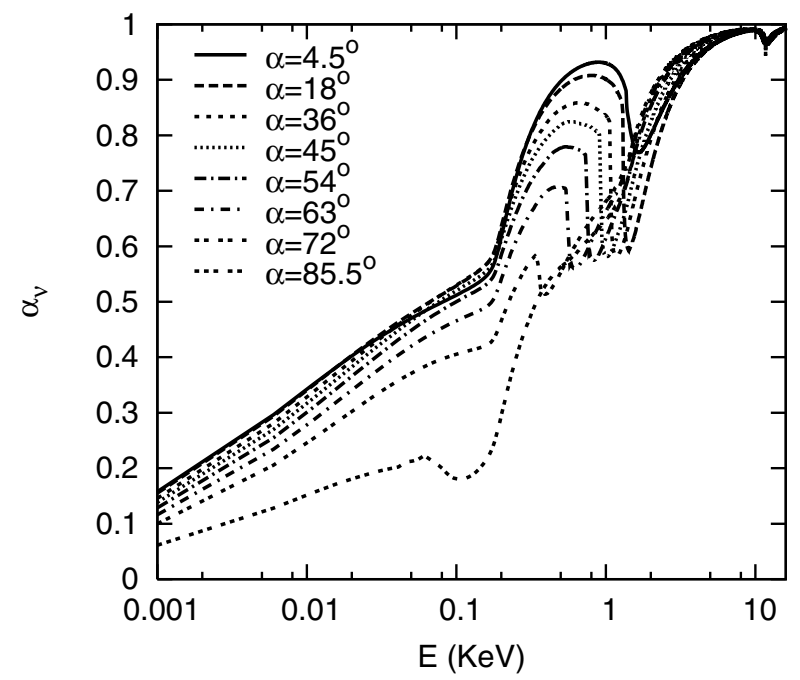

Fig. 3. Normalized emissivity integrated over all possible incident angles as a function of energy for $B=10^{12} \mathrm{G}$ and $T=10^{6} \mathrm{~K}$. Different orientations of the magnetic field are compared: from top to bottom $\theta=4.5,18,36,45,54,63,72$; and $85.5^{\circ}$.

the blackbody case for energies lower than $0.2-0.5 \mathrm{keV}$, depending on the incident angle. A reduction for energies close to that corresponding to the plasma frequency (indicated by the vertical line) is also evident in all cases. Notice how for incident angles close to $\pi / 2$ the emission is strongly suppressed in a wide range of energies.

Let us now comment on the variation of the emissivity with other relevant parameters. In Fig. 3 we show the normalized emissivity, integrated over all possible incident angles, as a function of the photon energy. We have taken $T=10^{6} \mathrm{~K}$ and $B=10^{12} \mathrm{G}$ and we show results for different magnetic field orientations ( $\alpha$ is the angle between the magnetic field and a vector normal to the surface). At energies greater than the electron plasma frequency (about $1 \mathrm{keV}$ in this case) the emitted flux approaches the $B B$ value $\left(\alpha_{v}=1\right)$, but the spectrum is significantly depressed at low energies. As explained 


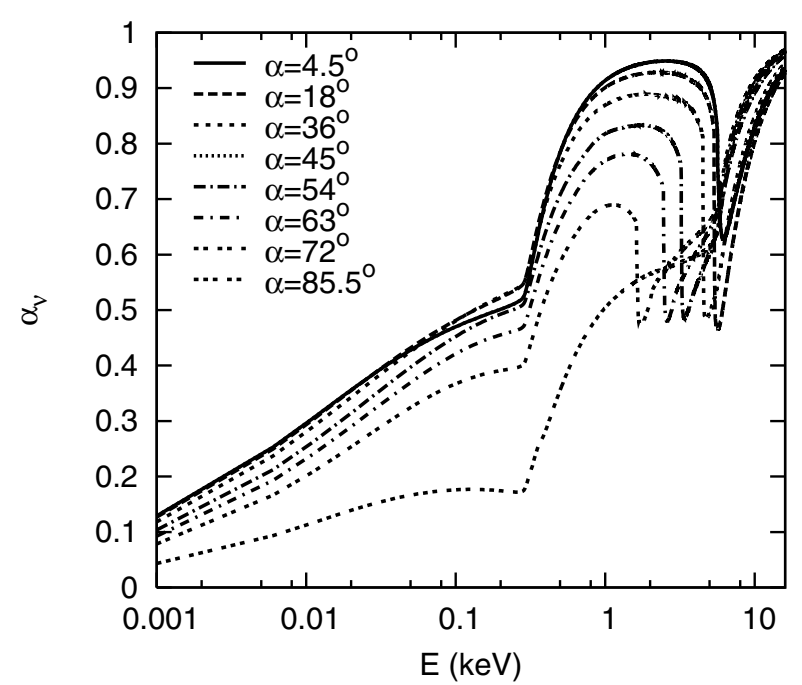

Fig. 4. Same as Fig. 3 but for $B=10^{13} \mathrm{G}$.

above, this is due to the fact that the refractive index has a large imaginary part. A resonance, which produces a reduction of the emissivity, is also visible at energies close to the electron plasma frequency. Notice that the resonant energy is not exactly the plasma frequency but depends on the angle between the magnetic field and the surface. This can be understood considering that the refractive index of one of the modes becomes infinity when the coefficient of $n^{4}$ in the dispersion relation vanishes. Again, neglecting damping for simplicity, we have $P+(S-P) \sin ^{2} \alpha=0$, which leads to

$\omega^{4}-\left(\omega_{B}^{2}+\omega_{\mathrm{p}}^{2}\right) \omega^{2}+\omega_{\mathrm{p}}^{2} \omega_{B}^{2} \cos ^{2} \alpha=0$.

The solution of this biquartic equation is

$\omega_{ \pm}^{2}=\frac{\omega_{B}^{2}+\omega_{\mathrm{p}}^{2}}{2}\left[1 \pm\left(1-\frac{4 \omega_{\mathrm{p}}^{2} \omega_{B}^{2} \cos ^{2} \alpha}{\left(\omega_{B}^{2}+\omega_{\mathrm{p}}^{2}\right)^{2}}\right)^{1 / 2}\right]$.

For magnetic fields perpendicular to the surface $\alpha=0$, we have $\omega_{+}^{2}=\omega_{B}^{2}$ and $\omega_{-}^{2}=\omega_{\mathrm{p}}^{2}$, but for magnetic fields parallel to the emitting surface one finds $\omega_{+}^{2}=\omega_{B}^{2}+\omega_{p}^{2}$ and $\omega_{-}^{2}=0$. Therefore, as we approach $\alpha=\pi / 2$, the resonance corresponding to the plasma frequency in the $\alpha=0$ case is shifted to lower and lower energies.

The same but for $B=10^{13} \mathrm{G}$ is shown in Fig. 4 . Qualitatively we obtain the same behaviour, but the plasma frequency is larger and the resonant energy is shifted consistently to higher values. Notice that the frequency below which the spectrum is depressed depends weakly on the magnetic field $\omega_{\mathrm{p}}^{2} / \omega_{B} \propto B^{1 / 5}$, and for standard values of the magnetic field falls in the range $0.1-0.2 \mathrm{keV}$. In this energy range the effect of interstellar medium absorption can make it difficult to distinguish between the two effects.

\subsection{The effect of motion of ions}

When we were finishing this paper, our attention was drawn to a very recent preprint (van Adelsberg et al. 2004) with similar results to ours. In this work, the authors included terms related to the motion of ions in the dielectric tensor, which result in

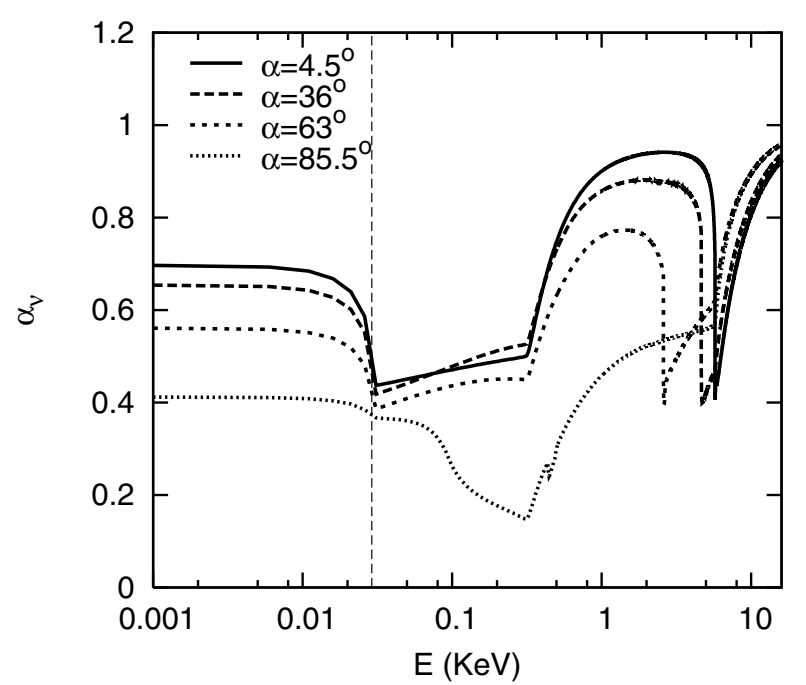

Fig. 5. Same as Fig. 4 but including the effect of the motion of ions. The vertical line corresponds to the ion cyclotron energy.

smaller reflectivity (larger emissivity) at frequencies below the ion cyclotron frequency $\left(\omega_{B_{i}}=\frac{Z e B}{m_{i} c}\right)$. The way in which the effect of ions is included is a crude simplification: as if they were free ions although they are in a lattice. It is quite doubtful that this approximation can actually represent reality, but it gives some interesting results that are an indication that more work is needed along that line. In short, free ions can be introduced in the calculations just by modifying the components of the dielectric tensor (8) in the following way (Ginzburg 1970; van Adelsberg et al. 2004):

$$
\begin{aligned}
\left(\begin{array}{l}
R \\
L
\end{array}\right) & =1-\frac{\omega_{\mathrm{p}}^{2}+\omega_{\mathrm{p}_{i}}^{2}}{\left(\omega \mp \omega_{B}\right)\left(\omega \pm \omega_{B_{i}}\right)+\mathrm{i} \omega v_{\perp}^{D} A^{\mp}} ; \\
P & =1-\frac{\omega_{\mathrm{p}}^{2}+\omega_{\mathrm{p}_{i}}^{2}}{\omega^{2}+\mathrm{i} \omega v_{\|}^{D}},
\end{aligned}
$$

where $\omega_{\mathrm{p}_{i}}=\left(4 \pi Z^{2} e^{2} n_{i} / m_{i}\right)^{1 / 2}$ is the ion plasma frequency and $A^{\mp}=1 \mp \frac{\omega_{B_{i}}}{\omega}\left(1-Z^{-1}\right)+\frac{m_{\mathrm{e}}}{m_{i}}$.

In Fig. 5 we show the normalized emissivity integrated over all incident angles, for $T=10^{6} \mathrm{~K}$ and $B=10^{13} \mathrm{G}$, including the effect of ions. The vertical line indicates the energy corresponding to the ion cyclotron frequency.

In order to understand the effect of ions we follow the same argument after Eq. (21). Neglecting damping for simplicity, and considering that the ion plasma frequency is much smaller than the electron plasma frequency $\left(\omega_{p_{i}} \ll \omega_{p}\right)$, the dispersion relation is simply

$n^{2}=\left(\begin{array}{l}L \\ R\end{array}\right)=1-\frac{\omega_{\mathrm{p}}^{2}}{\left(\omega \pm \omega_{B}\right)\left(\omega \mp \omega_{B_{i}}\right)}$.

When $\omega>\omega_{B_{i}}$ there is not much difference with respect to the case in which ions are not included. But when $\omega<\omega_{B_{i}}$, the new term in the denominator changes sign and the refractive index of the mode that acquired a large imaginary part when the ion contribution was not included becomes

$n^{2}=1+\frac{\omega_{p}^{2}}{\omega_{B} \omega_{B_{i}}}$

and the mode is no longer damped. 


\section{Spectral energy distribution}

The total spectral emission of the star, the specific luminosity, is obtained by integrating the specific intensity over the solid angle pointing outward and over the surface of the star,

$$
\begin{array}{r}
L_{v}=R^{2} \int_{0}^{2 \pi} \mathrm{d} \phi \int_{0}^{\pi} \sin \theta \mathrm{d} \theta \int_{0}^{2 \pi} \mathrm{d} \phi^{\prime} \\
\int_{0}^{\pi / 2} \mathrm{~d} \theta^{\prime} I_{v}\left(\theta, \phi, \theta^{\prime}, \phi^{\prime}\right) \cos \theta^{\prime} \sin \theta^{\prime}
\end{array}
$$

where $\theta$ and $\phi$ are the polar and azimuthal angles of a given point at the surface and $\theta^{\prime}$ and $\phi^{\prime}$ the polar and azimuthal angles of the direction defining the solid angle element.

The presence of the magnetic field, however, makes the emission anisotropic, and the observed specific flux $F_{v}$ is different from the integrated emitted flux obtained from the specific luminosity, i.e. $L_{v} / 4 \pi d^{2}$ for a star at a distance $d$. To calculate the observed specific flux we have to integrate only over the observed hemisphere $(\mathrm{OH})$. Denoting by $R$ the radius of the star,

$F_{v}=\frac{R^{2}}{d^{2}} \int_{\mathrm{OH}} I_{\nu}\left(\theta, \phi, \theta^{\prime}, \phi^{\prime}\right) \cos \theta^{\prime} \sin \theta \mathrm{d} \theta \mathrm{d} \phi$

where the angles $\theta^{\prime}$ and $\phi^{\prime}$ specify the angular direction from the surface element towards the observer. Note that in this paper we are neglecting general relativistic effects. For example, light bending increases the area of the observed hemisphere, depending on the compactness $(M / R)$ of the neutron star, as discussed in Page (1995) or Psaltis et al. (2000).

In order to get a more accurate feeling of what a realistic emission would be, in the following we will consider that the magnetic field has a dipolar geometry, this is

$B(R, \theta)=\frac{B_{\mathrm{p}}}{2}\left(1+3 \cos ^{2} \theta\right)^{1 / 2}$

with $B_{\mathrm{p}}$ being the intensity of the magnetic field at the pole.

The integrated spectrum as a function of the energy is shown in Fig. 6 for different intensities of the dipolar magnetic field and a temperature of $T=10^{6} \mathrm{~K}$. For reference, the $B B$ spectrum is also depicted (solid line). The broadband spectrum is essentially featureless and the flux is systematically lower than the $B B$ flux for the same temperature; this effect is more significant at lower energies. Notice that this flux must be understood as an average over the whole emitting surface, which is radiating anisotropically. The real observed flux depends on the particular location of the observer, as defined by Eq. (38). In Fig. 7 we show the flux observed from three different directions, forming an angle of $0^{\circ}, 45^{\circ}$, and $90^{\circ}$, with the polar axis, respectively. Near the maximum the differences between observers can be at most a factor 2, but the observer location becomes less relevant as we move towards either the low and high energy part of the spectrum.

At this point, the two main features of the "metallic surface" model seem to be: first, an almost featureless spectrum, and second, an overall flux smaller than that of a BB at the same temperature, especially at low energies. However, as we mentioned in the introduction, there is an important point to

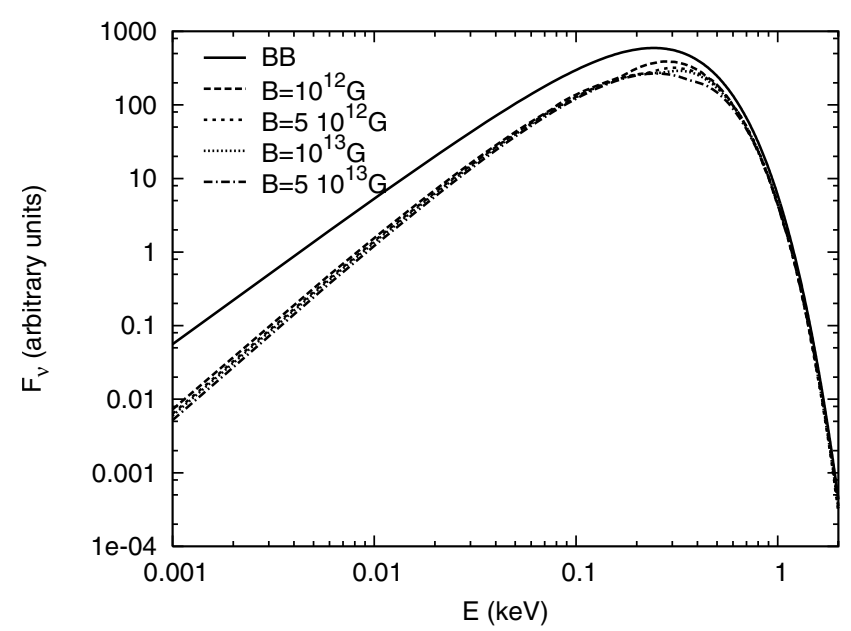

Fig. 6. Integrated emitted flux, $L_{v} / 4 \pi D^{2}$, (in arbitrary units) for a uniform temperature of $T=10^{6} \mathrm{~K}$. The magnetic field geometry has been taken to be a dipolar distribution and several values of the magnetic field strength are compared: $B_{\mathrm{p}}=10^{12}, 5 \times 10^{12}, 10^{13}$, and $5 \times 10^{13} \mathrm{G}$ (values at the magnetic pole).

consider. The fact that the condensed surface is strongly magnetized makes the thermal conductivity very different in the directions perpendicular and parallel to the magnetic field lines. Similar effects have been pointed out to be relevant in the envelope (Greenstein \& Hartke 1983; Page 1995) or in the crust, where a very recent study (Geppert et al. 2004), finds that the anisotropy in the temperature distribution depends very strongly on the particular geometry of the internal magnetic field, resulting in variations of temperature of up to a factor 5 . In a separate work (Pérez-Azorín et al. 2005), we will report results from a detailed study of the temperature distribution obtained from 2D diffusion calculations for different magnetic field geometries. For the purpose of understanding qualitatively the effects on the observed spectrum, in this paper we will limit our analysis to the case in which the temperature distribution has the following angular dependence

$T=T_{\mathrm{p}}\left[\cos ^{2} \theta_{B}+\chi \sin ^{2} \theta_{B}\right]^{1 / 4}$,

where $\theta_{B}$ is the angle between the field and the normal to the surface, $\chi$ is the ratio between the thermal conductivities normal and parallel to the magnetic field, and $T_{\mathrm{p}}$ is the polar temperature (where $\theta_{B}=0$ ). The origin of this distribution has been discussed in previous works on neutron star envelopes (Greenstein \& Hartke 1983; Page 1995). Note that for a dipolar magnetic field $\chi$ is a function of the polar angle because the magnetic field strength varies with the latitude.

In Fig. 8 we show the same three cases as in Fig. 7 but for the anisotropic temperature distribution given by Eq. (40) with $T_{\mathrm{p}}=10^{6} \mathrm{~K}$. The optical band of the spectrum is not very much altered, but the high energy tail is significantly depressed. This effect, combined with the low energy depression caused by the high reflectivity of the metallic surface at low energies, results in a broadband spectrum that mimics the $B B$ spectrum but with an overall reduced flux by nearly a factor of 10 . This means that, for a fixed distance to the source, the observed flux from such a particular neutron star surface will look like a Planckian 


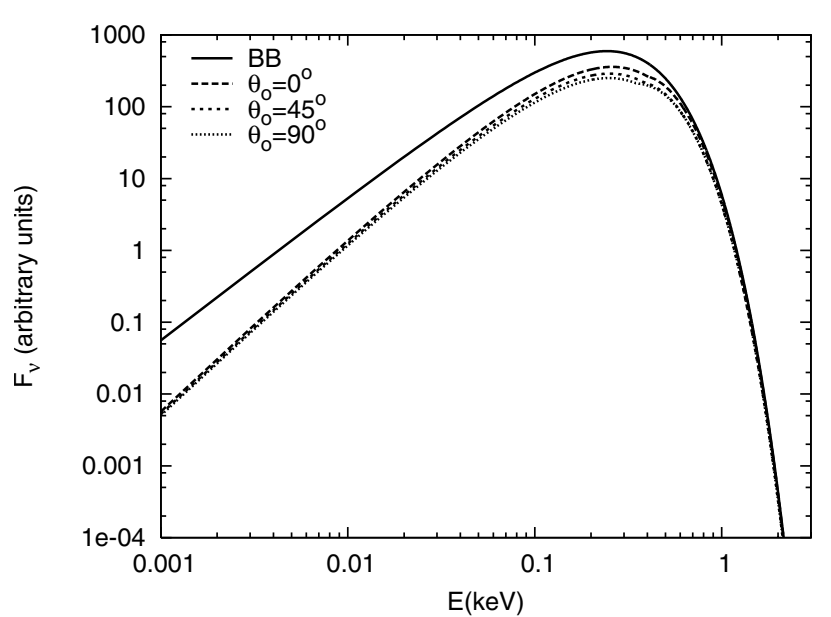

Fig. 7. Observed flux, $F_{v}$, (in arbitrary units) for a uniform temperature of $T=10^{6} \mathrm{~K}$ and three different observation angles. The magnetic field geometry has been taken to be a dipolar distribution with $B_{\mathrm{p}}=$ $5 \times 10^{13} \mathrm{G}$.

spectrum, but the apparent area of the source (and therefore the radius) would be underestimated by a large factor. To make this point more explicit, in Fig. 9 we plot the observed flux (dashes) from a model with $B_{\mathrm{p}}=5 \times 10^{13}, T_{\mathrm{p}}=10^{6}$ and $\theta_{o}=90^{\circ}$, as seen after taking into account interstellar medium absorption with $n_{\mathrm{H}}=1.4 \times 10^{20} \mathrm{~cm}^{-2}$, compared with a uniform temperature, blackbody model that fits the X-ray part of the spectrum (solid line). The parameters of the BB model are: $T=10^{6} \mathrm{~K}, n_{\mathrm{H}}=1.3 \times 10^{20} \mathrm{~cm}^{-2}$, and a relative normalization factor $\left(\propto\left(R_{\infty} / d\right)^{2}\right)$ of $1 / 5$. Consequently, the apparent estimated value of the $R_{\infty} / d$ is 2.3 times lower than that of the "real" model, despite the X-ray spectrum being very similar.

For comparison, we have also included the effects of ions in Fig. 9 (dotted line). Notice that the optical flux of this model is a factor 3 larger than the BB fit prediction, similarly to what has been observed in isolated neutron stars. Nevertheless, it must be stressed that this is just a crude approximation, with no pretension of being the real answer to the observed optical excess, but it serves to illustrate how important it is to understand details about the magnetic field structure, the properties of the solid lattice, and the temperature distribution, before one is able to make robust estimates of the neutron star properties (e.g. radius).

\section{Final remarks}

In this paper we have revisited the bare neutron star surface model first studied in detail in B80, which in the last years is becoming popular again and attracting the attention of other groups (Lai 2001; Turolla et al. 2004; van Adelsberg et al. 2004). Our results for constant temperature magnetized surfaces confirm qualitatively those reported earlier by other authors, with small quantitative differences due to the fact that in previous works (Turolla et al. 2004) some approximations (neglecting one mode) were made, which made the results dependent on the cutoff value of the imaginary part of the refraction index. In general, models with uniform temperature show a broadband spectrum that is very close to

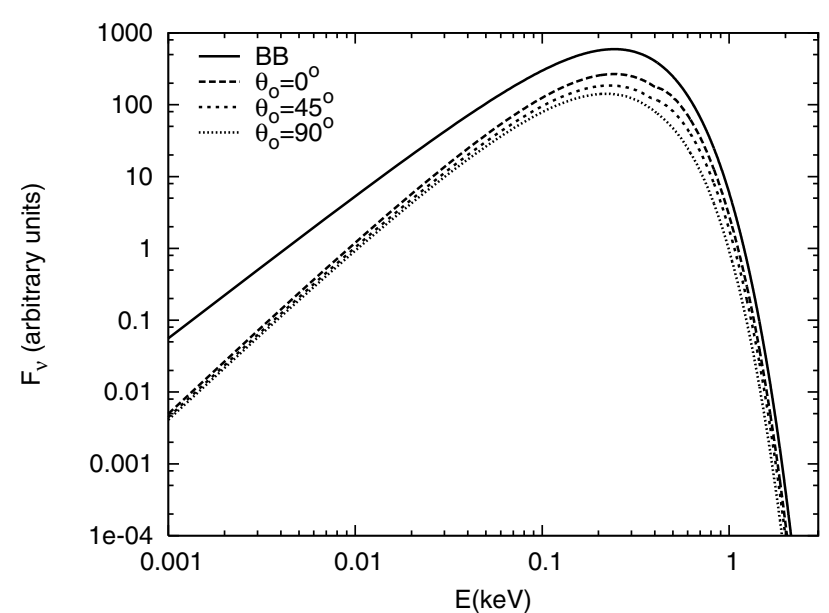

Fig. 8. Observed flux, $F_{v}$, (in arbitrary units) for an anisotropic temperature distribution described in Eq. (40) with $T_{\mathrm{p}}=10^{6} \mathrm{~K}$ and three different observation angles. The magnetic field geometry has been taken to be a dipole with $B_{\mathrm{p}}=5 \times 10^{13} \mathrm{G}$. The corresponding Planckian spectrum for $T=10^{6} \mathrm{~K}$ is also shown for comparison.

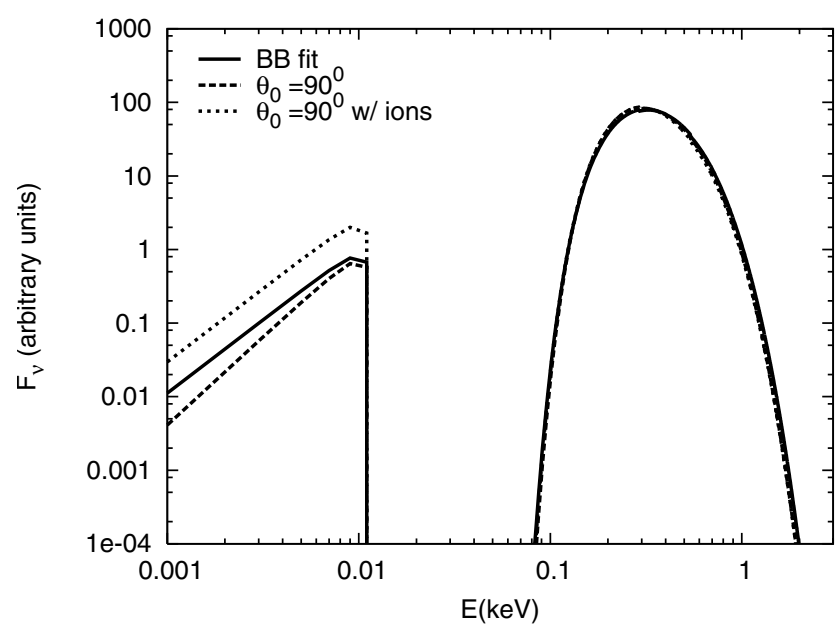

Fig. 9. Observed flux, $F_{v}$, (in arbitrary units) for the anisotropic temperature distribution described in Eq. (40) with $T_{\mathrm{p}}=10^{6} \mathrm{~K}$ and $\theta_{o}=90^{\circ}$, as it would be seen after taking into account interstellar medium absorption with $n_{\mathrm{H}}=1.4 \times 10^{20} \mathrm{~cm}^{-2}$. We show results with (dots) and without (dashes) including the effect of ions. A uniform temperature, blackbody fit of the X-ray part of the spectrum is also depicted with solid lines $\left(T=10^{6} \mathrm{~K}, n_{\mathrm{H}}=1.3 \times 10^{20} \mathrm{~cm}^{-2}\right)$ but corrected by a factor $1 / 5$, that is, the apparent estimated value of the $R_{\infty} / d$ is 2.23 times lower than that of the "real" model.

Planckian at energies above $\omega_{\mathrm{p}}^{2} / \omega_{B}$, and significantly depressed (up to a factor 10) in the optical band. The spectrum is almost featureless, with only some small bumps at energies where the interstellar medium absorption makes it difficult to distinguis and fine tune between different parameters. However, in our opinion, there is a key point that is barely addressed in previous works and needs more attention: in the crust and the condensed outer layer the assumption of a homogeneous temperature distribution is inconsistent because magnetic fields of the order of or larger than $10^{13} \mathrm{G}$ imply some degree of anisotropy in the thermal conductivity. The analogous effect in the inner crust has recently been studied (Geppert et al. 2004), finding large variations of temperature when the magnetic field is confined 
to the crust. Notice that, if in the inner regions we have superconducting protons, as seems to be the case, this situation is very likely. A detailed analysis of the realistic, self-consistent emission from bare neutron star surfaces requires, therefore, multidimensional transport calculations with the presence of magnetic fields, and using appropriate boundary conditions (accordingly with the calculated $\alpha_{v}$ ). Such calculations, as well as fits to real data, are in progress (Pérez-Azorín et al. 2005) and will be reported elsewhere. In the mean time, one can guess what sort of changes to expect by looking at the emitted spectrum produced by an ad-hoc temperature distribution, as we discussed in this paper. This example was very illustrative of one fact: the observed flux of such an object is very close to a $B B$ spectrum, but we might be underestimating the area of the emitter (and therefore its size) by a large factor. In addition, depending on the strength of the magnetic field, and including the effects of ions, we could even obtain an optical flux larger (relative to the BB case) than that in the X-ray band, which is commonly found in all isolated neutron stars with an optical counterpart.

As stated before, general relativistic effects have not been included in this work. A first correction is simply to redshift all energies and temperatures. This is of crucial importance if spectral features are present, but only translates into an overall scale factor if we consider a BB or a featureless spectrum. A second effect might be more relevant. Light bending increases the observed emitting area, smearing out partially the differences between different observers, depending on how compact the object is. In order to make precise parameter estimates at least these two major corrections should be included. In summary, there is much physics to be understood and analyzed in the near future before drawing robust conclusions on the nature of isolated compact objects (neutron stars vs. strange stars), and measuring with precision their radii and masses.

Acknowledgements. This work has been supported by the Spanish Ministerio de Ciencia y Tecnología grant AYA 2001-3490-C02. J.A.P. is supported by a Ramón y Cajal contract from the Spanish MCyT. We thank Fred Walter, Jim Lattimer and Dany Page for interesting comments and discussions.

\section{Appendix A: Reflected wave amplitudes}

The system of Eq. (24) can be solved for the electric field of the reflected wave in terms of the incident one. The result reads as follows:

$$
\begin{aligned}
E_{\perp}^{\prime \prime}= & \mathcal{D}^{-1}\left\{\left[B_{1}\left(1-w_{1}\right)\left(A_{2} \sin i+C_{2} \cos i\right)\right.\right. \\
& \left.-\quad B_{2}\left(1-w_{2}\right)\left(A_{1} \sin i+C_{1} \cos i\right)\right] E_{\perp} \\
& \left.+2 B_{1} B_{2}\left(w_{1}-w_{2}\right) \sin i \cos i E_{\|}\right\} \\
E_{\|}^{\prime \prime}= & \mathcal{D}^{-1}\left\{2\left(A_{1} C_{2}-A_{2} C_{1}\right) E_{\perp}\right. \\
+ & {\left[B_{1}\left(1+w_{1}\right)\left(A_{2} \sin i-C_{2} \cos i\right)\right.} \\
& \left.\left.-B_{2}\left(1+w_{2}\right)\left(A_{1} \sin i-C_{1} \cos i\right)\right] E_{\|}\right\}
\end{aligned}
$$

where

$$
\begin{aligned}
w_{\mathrm{m}} & =\frac{\sqrt{n_{\mathrm{m}}^{2}-\sin ^{2} i}}{\cos i}, \\
\mathcal{D} & =B_{1}\left(1+w_{1}\right)\left(A_{2} \sin i+C_{2} \cos i\right) \\
& -B_{2}\left(1+w_{2}\right)\left(A_{1} \sin i+C_{1} \cos i\right) .
\end{aligned}
$$

From these equations the original expressions in B80 can be recovered by using the relation

$C_{\mathrm{m}}=\sin ^{2} i+A_{\mathrm{m}} w_{\mathrm{m}} \sin i \cos i$.

We have found a typo in the first line of Eq. (19) in B80, where the last $\mathcal{A}_{2}$ should be a $\mathcal{B}_{2}$. Notice that Turolla et al. (2004) expressed the amplitudes of the reflected waves in a different way. The reason for this apparent difference is the same that led to different expressions for the coefficient $a_{\mathrm{m}}$ defined by Eq. (22): they used a different linear combination of the three equations arising from Eq. (15). We have checked numerically that both formulations are equivalent when $n_{\mathrm{m}}$ is a root of Eq. (20).

\section{References}

Burwitz, V., Zavlin, V. E., Neuhäuser, R., et al. 2001, A\&A, 379, L35

Burwitz, V., Haberl, F., Neuhäuser, R., et al., 2003, A\&A, 399, 1109

Brinkmann, W. 1980, A\&A, 82, 352352

Cordes, J. M., Romani, R. W., \& Lundgren, S. C. 1993, Nature, 362, 133

Drake, J. J., Marshall, H. L., Dreizla, S., et al. 2002, ApJ, 572, 996

Geppert, U., Küker, M., \& Page, D. 2004, A\&A, 426, 267

Ginzburg, V. L. 1970, in Propagation of electromagnetic waves in plasmas, 2nd ed. (Oxford: Pergamon press)

Greenstein, G., \& Hartke, G. J. 1983, ApJ, 271, 283

Hernquist, L. 1984, ApJS, 56, 325

Kulkarni, S. R., \& Hester, J. J. 1988, Nature, 335, 801

Lai, D. 2001, Rev. Mod. Phys. 73, 629

Lattimer, J. M., \& Prakash, M. 2001, ApJ 550, 426

Miller, M. C. 1992, MNRAS, 255, 129

Neuhaüser, R., Thomas, H. C., \& Walter, F. M. 1998, The Messenger, 92,27

Page, D. 1995, ApJ. 442, 273

Pavlov, G. G., Zavlin, V. E., Truemper, J., \& Neuhauser, R. 1996, ApJ 472, L33

Pérez-Azorín, J. F., Miralles J. A., \& Pons J. A. 2005, in preparation

Pons, J. A, Walter, F. M., Lattimer, J. M., et al. 2002, ApJ, 564, 981

Potekhin, A. Y. 1999, A\&A 351, 787

Psaltis, D., Özel, F., \& DeDeo, S. 2000, ApJ, 544, 390

Rajagopal, M., \& Romani, R. W. 1996, ApJ, 461, 327

Rajagopal, M., Romani, R. W., \& Miller, M. C. 1997, ApJ, 479, 347

Romani, R. W. 1987, ApJ, 313, 718

Turolla, R., Zane, S., \& Drake, J. J. 2004, ApJ, 603, 265

van Adelsberg, M., Lai, D., \& Potekhin, A. 2004 [arXiv:astro-ph/0406001]

van Kerkwijk, M. \& Kulkarni, S. 2000, ESO press release PR 19/00

Walter, F. M., Wolk, S. J., \& Neuhäuser, R. 1996, Nature, 379, 233

Walter, F. M., \& Matthews, L. D. 1997, Nature, 389, 358

Walter, F. M., \& Lattimer, J. M. 2002, ApJ, 575, L145

Zavlin, V. E., Pavlov, G. G., Shibanov, Y. A., \& Ventura, J. 1995, A\&A, 297, 441 